\title{
Landau-Pomeranchuk-Migdal effect in thermal field theory
}

\author{
P. Aurenche ${ }^{(1)}$, F. Gelis ${ }^{(2)}$, H. Zaraket ${ }^{(1)}$
}

May 28, 2018

1. Laboratoire de Physique Théorique LAPTH, UMR 5108 du CNRS, associée à l'Université de Savoie, BP110, F-74941, Annecy le Vieux Cedex, France

2. Brookhaven National Laboratory, Physics Department, Nuclear Theory, Upton, NY-11973, USA

\begin{abstract}
In recent studies, the production rate of photons or lepton pairs by a quark gluon plasma has been found to be enhanced due to collinear singularities. This enhancement pattern is very dependent on rather strict collinearity conditions between the photon and the quark momenta. It was estimated by neglecting the collisional width of quasi-particles. In this paper, we study the modifications of this collinear enhancement when we take into account the possibility for the quarks to have a finite mean free path. Assuming a mean free path of order $\left(g^{2} T \ln (1 / g)\right)^{-1}$, we find that only low invariant mass photons are affected. The region where collision effects are important can be interpreted as the region where the LandauPomeranchuk-Migdal effect plays a role in thermal photon production by bremsstrahlung. It is found that this effect modifies the spectrum of very energetic photons as well. Based on these results and on a previous work on infrared singularities, we end this paper by a reasonable physical picture for photon production by a quark gluon plasma, that should be useful to set directions for future technical developments.
\end{abstract}

LAPTH-790/2000, BNL-NT-00/11 


\section{Introduction}

The photon production rate is thought to be a quantity of phenomenological interest in heavy ions collisions, possibly enabling one to detect the formation of a quark-gluon plasma. Part of the interest in this electromagnetic observable comes from the fact that photons are relatively weakly coupled to nuclear matter $\left(\alpha_{E M} \ll \alpha_{S}\right)$. Given the typical size of the system in such collisions (much smaller than the mean free path of a photon), they do not re-interact between their production and their observation. As a consequence, photons (real photons, or virtual photons decaying eventually into a lepton pair) can provide information on the state of the system at the time they were produced.

In order to calculate the photon yield from a hot quark-gluon plasma, thermal field theory is the tool of choice since its Feynman rules automatically take into account the presence of a thermal bath with the appropriate distributions of partons. Thermal gauge theories are however plagued by infrared singularities arising from the Bose-Einstein distribution functions which are singular at zero energy. An improvement over the bare Feynman rules is achieved by the resummation of one-loop thermal contributions known as hard thermal loops (HTL) 1, 2, 3, 4, 5. 5. These thermal corrections make it possible to include in the propagators effects like Debye screening or Landau damping, and transform partons into massive quasi-particles. From a quantitative perspective, they provide important changes to the dynamics of soft modes (of momentum of order $g T$ or less). This resummation lacks however two features: it does not provide any Debye screening for static magnetic fields (such a screening is expected to arise non-perturbatively in QCD at the length scale $\left(g^{2} T\right)^{-1}$ ), and its quasiparticles do not undergo collisions (their collisional] width is also a sub-leading effect of order $\left.g^{2} T \ln (1 / g)\right)$.

In the framework of thermal field theory, the photon/dilepton rate is obtained via the calculation of the imaginary part of the retarded photon polarization tensor 6, 77. This object has been evaluated at one-loop in the HTL-improved perturbative expansion, both for virtual 8, 9] and real photons 110, 11, 12, 13, 14]. More recently, new processes like bremsstrahlung were studied in detail in this framework and have been found to be dominant sources of low invariant mass photons 15, 16, 17]. Despite the fact that this process arrives only in 2-loop contributions to the photon polarization tensor, it is always important because of a strong collinear enhancement. Indeed, it was found in [16] that for a very small photon invariant mass, the corresponding diagram contains collinear singularities that, when regularized by a thermal quark mass of order $g T$, give an enhancement by a factor of order $1 / g^{2}$ over naive estimates coming from power counting.

\footnotetext{
${ }^{1}$ In a plasma, it is very important to distinguish two contributions to the total width: the decay width made of the zero temperature contribution to the imaginary part of the quark self-energy, and the collisional width which exists only in a medium. The latter is also called "anomalous damping rate" (or just "damping rate" for short) and is of order $g^{2} T \ln (1 / g)$, while in QED/QCD the former starts at the order $g^{4}$. The width $\Gamma$ that we introduce in this paper is the collisional width, and its inverse is the mean free path of the quark in the plasma.
} 
After finding that certain 2-loop terms are contributing at leading order, one may wonder whether this result is specific to this 2-loop contribution only or if, on the contrary, this is an indication of the breakdown of perturbative expansion (even improved with HTLs). In a recent paper [18], we studied what is the effect of loop corrections to this 2-loop diagram. Power counting indeed indicates a problem very similar to the problem raised by Linde [19] for the calculation of the free energy, due to the lack of Debye screening for static magnetic modes. This problem is avoided for the production of massive enough photons, because some cancellations (occuring within any given topology, when one is summing over all the cuts contributing to the imaginary part) generate a kinematical cutoff large enough to prevent any sensitivity to the non-perturbative scale $g^{2} T$. Unfortunately, this cutoff is smaller than $g^{2} T$ whenever the invariant mass of the produced photon is too small (typically $Q^{2}<g^{2} T q_{0}$ for $q_{0}<T$ ). In this low invariant mass region, the photon rate is therefore non perturbative: exchanged transverse gluons reach the scale $g^{2} T$ of the non-perturbative "magnetic mass", and an infinite set of diagrams must be resummed.

In this paper, we present a completely different approach to higher order corrections, that completes the picture outlined in 18. The idea behind the present study is that a width on the quark propagator will act as a regulator in the collinear sector, because it moves the poles of the propagator away from the real energy axis. Such a collisional width is necessarily a higher-loop effect, because the hard thermal loop framework does not take into account the collisions of quasi-particles. Having in mind the fact that 2-loop contributions are important because of collinear enhancement, an important question to answer is how much of this enhancement is lost when an additional regulator like a width is taken into account. This is the question we want to address in this paper, by calculating the same 2-loop diagrams as in 15, 16], in the presence of a quark width. To be more definite, and keep the model as well as the calculations simple, we use a momentum-independent width.

A word of caution is necessary here: the formulae found using this simple model should not be taken as an accurate quantitative account of what the effect of such a width will be on thermal photon production rates. Indeed, the constant width model is probably too naive to be realistic, and more importantly our calculation disregards the fact that a modification of the vertices should in principle accompany the modification of the quark propagators. Nevertheless, this simple approach is sufficient here for our purpose which is just to determine the region in which effects of a width of order $g^{2} T \ln (1 / g)$ are to be expected, since that gives another handle on how and when higher order corrections may affect photon production by a quark-gluon plasma.

We find that the region where a width of order $g^{2} T \ln (1 / g)$ is important is very similar to the one found in [18] for the contribution of higher-loop topologies due to an IR sensitivity to the scale of the magnetic mass. Despite their similarity, these two non perturbative regions have different physical interpretations. In particular, we find that the sensitivity to the collisional width of the quarks is a manifestation of the Landau-Pomeranchuk-Migdal effect. Indeed, it occurs when the formation time of the photon is larger than the mean free path 
of the quark producing the photon. An interesting consequence of our study is that the LPM effect also modifies the spectrum of highly energetic photons.

The structure of the paper is as follows. Section 2 makes more precise our modeling of the mean free path for the quarks. In section 3, we start by computing the 1-loop contribution in presence of a width. Although not related to collinear singularities in any way, the purpose of this warmup exercise is twofold: it illustrates the technology (how one does calculations with a width), and it shows in a simple way how collisions can open up the phase space. We also show that this 1-loop contribution is canceled by the resummation of vertex corrections, and is therefore not physical.

In section 4, we present the 2-loop calculation with a quark width, and obtain a rather simple generalization of the formulae of [16. These formulae are discussed extensively in section 5 , in which we also determine the region where a width of order $g^{2} T \ln (1 / g)$ plays an important role.

Section 6 is devoted to establishing the connection between the previous results and the LPM effect. In section 7 , we study the process obtained from bremsstrahlung by crossing symmetry, which turns out to be important in the region of large photon energy. We show that this process is also affected by the LPM effect. The last section contains concluding remarks. In particular, we combine the present work on the LPM effect with previous results on infrared singularities 18] in order to make a syntesis and present a reasonable physical picture of thermal photon production.

\section{Model}

Let us first make our framework more definite. The only modification compared to the context extensively described in [16] is that the quark propagators are given a width, as outlined by the following substitution in the retarded and advanced propagators:

$$
\Delta_{R, A}(P) \equiv \frac{1}{P^{2}-M_{\infty}^{2} \pm i p_{0} \epsilon} \rightarrow \boldsymbol{\Delta}_{R, A}(P) \equiv \frac{1}{\left(p_{0} \pm i \Gamma\right)^{2}-\boldsymbol{p}^{2}-M_{\infty}^{2}},
$$

where $M_{\infty} \sim g T$ is the usual asymptotic thermal mass for hard quarks [20, 16], and where $\Gamma$ is a constant width. The expression in Eq. (1) is sufficient for the physics we consider, which is dominated by hard quark momenta close to the mass shell. Whenever we need to estimate the order of magnitude of a term containing $\Gamma$ in the following, we assume that it is of order $g^{2} T \ln (1 / g)$ (with $g \ll 1)$. Note that we could as well have written

$$
\boldsymbol{\Delta}_{R, A}(P)=\frac{1}{P^{2}-M_{\infty}^{2} \pm 2 i p_{0} \Gamma}
$$

since the two differ by the small correction $\Gamma^{2} \ll M_{\infty}^{2}$ to the real part of the denominator. The retarded (resp. advanced) propagator has two complex poles in the $p_{0}$ plane, located at $p_{0}= \pm \omega_{p}-i \Gamma\left(\right.$ resp. $\left.p_{0}= \pm \omega_{p}+i \Gamma\right)$, with $\omega_{p} \equiv$ $\sqrt{ }\left(\boldsymbol{p}^{2}+M_{\infty}^{2}\right)$. 


\section{1-loop study}

\subsection{Calculation}

Let us now consider the simplest calculation conceivable in this framework, namely the computation of the 1-loop contribution to the photon polarization tensor. Our purpose is simply to illustrate possible changes brought in when taking into account a width for the quarks running in the loop. We do not attempt a complete calculation including HTL vertices, as pictured on Fig. 11. The

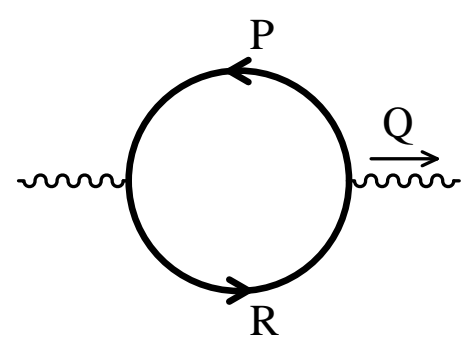

Figure 1: 1-loop diagram. The boldface quark line denotes the resummation of a width on the fermion propagator.

contribution of this diagram to the imaginary part of the retarded polarization tensor is trivial to obtain:

$$
\begin{aligned}
\operatorname{Im} \Pi_{\mu}^{\mu}(Q) \approx \frac{1}{2} e^{2} & \int \frac{d^{4} P}{(2 \pi)^{4}}\left[n_{F}\left(r_{0}\right)-n_{F}\left(p_{0}\right)\right] \operatorname{Tr} \\
& \times\left[\boldsymbol{\Delta}_{R}(P)-\boldsymbol{\Delta}_{A}(P)\right]\left[\boldsymbol{\Delta}_{R}(R)-\boldsymbol{\Delta}_{A}(R)\right],
\end{aligned}
$$

where $\operatorname{Tr}$ denotes the Dirac's trace associated to the quark loop:

$$
\operatorname{Tr}=-8 P \cdot R .
$$

The case with $\Gamma=0$ is made simple by the fact that the differences $\boldsymbol{\Delta}_{R}-\boldsymbol{\Delta}_{A}$ are $\delta$ functions, thereby enabling some of the integrals to be performed trivially. Let us remind that for the case $Q^{2}<4 M_{\infty}^{2}$ which we assume throughout this paper, $\operatorname{Im} \Pi^{\mu}{ }_{\mu}(Q)=0$ if $\Gamma=0$ due to incompatibilities between the $\delta$ functions. To evaluate Eq. (3) when $\Gamma \neq 0$, we perform the integral over $p_{0}$ by closing the real axis in the complex energy plane and using the theorem of residues.

Note first that one can disregard the poles of the statistical weights $n_{F}\left(p_{0}\right)-$ $n_{F}\left(r_{0}\right)$. Indeed, these poles are the imaginary fermionic Matsubara frequencies and are to be plugged into differences like $\boldsymbol{\Delta}_{R}(P)-\boldsymbol{\Delta}_{A}(P)$. The fact that $\Gamma \ll T$ makes these differences very small 2 . In other words, the only important

\footnotetext{
${ }^{2}$ Strictly speaking, these contributions are needed to ensure that the final result is a real number. Indeed, when one picks poles like $p_{0}=\omega_{p}+i \Gamma$ from the propagators and plugs them in the distribution functions, the latter become complex numbers. Their (small) imaginary
} 
terms are those for which denominators like $P^{2}-M_{\infty}^{2}$ are small, and comparable to $p_{0} \Gamma$. This cannot happen if $p_{0}$ is an imaginary number of order $T$.

We are therefore left with the poles of the propagators themselves. At this point, we obtain the following result:

$$
\begin{aligned}
\operatorname{Im} \Pi_{\mu}^{\mu}(Q) & \approx \frac{1}{2} e^{2} \int \frac{d^{3} \boldsymbol{p}}{(2 \pi)^{3}} \sum_{\eta= \pm 1}\left[n_{F}\left(q_{0}+\eta \omega_{p}\right)-n_{F}\left(\eta \omega_{p}\right)\right] \operatorname{Tr}\left(p_{0}=\eta \omega_{p}\right) \\
& \times \frac{\eta}{2 \omega_{p}}\left[\frac{1}{\left(q_{0}+\eta \omega_{p}+2 i \Gamma\right)^{2}-\omega_{r}^{2}}-\frac{1}{\left(q_{0}+\eta \omega_{p}-2 i \Gamma\right)^{2}-\omega_{\boldsymbol{r}}^{2}}\right]
\end{aligned}
$$

We can also note for later use that the result of this approximation could have been obtained by starting from an expression slightly different from Eq. (3):

$$
\begin{aligned}
\operatorname{Im} \Pi_{\mu}^{\mu}{ }_{\mu}(Q) \approx \frac{1}{2} e^{2} & \int \frac{d^{4} P}{(2 \pi)^{4}}\left[n_{F}\left(r_{0}\right)-n_{F}\left(p_{0}\right)\right] \operatorname{Tr} \\
& \times 2 \pi \epsilon\left(p_{0}\right) \delta\left(P^{2}-M_{\infty}^{2}\right)\left[\boldsymbol{\Delta}_{R}^{2 \Gamma}(R)-\boldsymbol{\Delta}_{A}^{2 \Gamma}(R)\right],
\end{aligned}
$$

where we denote

$$
\boldsymbol{\Delta}_{R, A}^{2 \Gamma}(R) \equiv \frac{1}{\left(r_{0} \pm 2 i \Gamma\right)^{2}-\boldsymbol{r}^{2}-M_{\infty}^{2}} .
$$

In other words, if $\Gamma \ll T$, then we can as well put twice the width on one of the two quark lines, and nothing in the other quark line ${ }^{3}$.

After Eq. (5), the angular integration over $d \Omega_{p}$ is trivial. In the case where $\eta=+1$ (process $q \rightarrow q \gamma$ ), we find:

$$
\begin{aligned}
\operatorname{Im} \Pi_{\mu}^{\mu}(Q) \approx \frac{e^{2} \Gamma}{2 \pi^{2} q_{0}} \int_{0}^{+\infty} & d p\left(q_{0}+p\right)\left[n_{F}\left(q_{0}+p\right)-n_{F}(p)\right] \\
& \times \ln \left(\frac{\left(\omega_{p}+p\right)^{2} q^{2}+\Gamma^{2}\left(q_{0}+p\right)^{2}}{\left(\omega_{p}-p\right)^{2} q^{2}+\Gamma^{2}\left(q_{0}+p\right)^{2}}\right) .
\end{aligned}
$$

In particular, assuming for the sake of simplicity that $q \approx q_{0}$, it is trivial to obtain the following asymptotic behaviors for soft and hard photons:

$$
\begin{array}{ll}
\text { If } q_{0} \ll T, & \operatorname{Im} \Pi^{\mu}{ }_{\mu}(Q) \sim e^{2} \Gamma T \ln \left(1+4 q_{0}^{2} / \Gamma^{2}\right), \\
\text { If } q_{0} \gg T, & \operatorname{Im} \Pi^{\mu}{ }_{\mu}(Q) \sim e^{2} \Gamma T \ln \left(T^{2} / \Gamma^{2}\right) .
\end{array}
$$

The main point is that these contributions are proportional to the width and vanish when $\Gamma=0$, in agreement with a direct calculation. As a side remark, one may note that for $\Gamma \sim g^{2} T \ln (1 / g)$ and soft photons $\left(q_{0} \sim g T\right)$, Eq. (9) is

part is canceled by the (small) contribution coming from the poles of $n_{F}\left(r_{0}\right)-n_{F}\left(p_{0}\right)$. For this approximation to be consistent, one must also neglect $i \Gamma$ in the hard argument of statistical weights.

${ }^{3}$ This is where it is important to have a constant width. Indeed, some intermediate step relies on the cancellation $\Gamma(P)-\Gamma(R)=0$. 
larger by a factor $1 / g$ than the 1-loop HTL result, while in the regime of Eq. (10) it is of the same order. It is the collision partners of the quarks that open up the phase-space (hard quarks colliding in the medium can emit a photon, a process forbidden for collisionless quarks) and make these contributions so large.

Another property of this result is that there is a suppression if $q_{0} \ll T$, such that $\operatorname{Im} \Pi_{\mu}^{\mu}(Q)$ tends to 0 when $q_{0} \rightarrow 0$. Equations similar to Eqs. (9) and (10) can be obtained when considering the contribution $\eta=-1$ to Eq. (6).

\subsection{Cancellation with vertex corrections}

This suppression was interpreted in [21] as the manifestation of the LPM effect. However, despite the suppression at small $q_{0}$, the connection with the LPM effect is not clear in this context. Indeed, the LPM effect is expected when the photon formation time is larger than the quark mean free path, a condition which never appears in [21], nor in the above 1-loop calculation. In fact, following [22, 23], we know that the propagator of a quark close to its mass shell on which a width is resummed can be evaluated by an eikonal resummation of the multiple collisions. By the same method, one can include in this resummation all the vertex corrections. Indeed, one can check that the photon polarization tensor at this level of approximation is proportional to (in space-time coordinates)

$$
\Pi^{\mu \nu}(x, y)=e^{2} \int\left[d A^{\mu}\right] e^{i S[A]} \operatorname{Tr}\left[\gamma^{\mu} S(x, y \mid A) \gamma^{\nu} S(y, x \mid A)\right],
$$

where $S[A]$ is the action of the gauge fields , and where $S(x, y \mid A)$ is the propagator of a quark in the background field $A$. This formula includes only one quark loop (in addition to the quark loops that may have been resummed in the gluon propagators and vertices in $S[A]$ ), and all orders in the gluon fields. In the eikonal approximation, the quark propagator has a very simple dependence on the field $A_{\mu}$ (which is a matrix $T^{a} A_{\mu}^{a}$ in QCD):

$$
S(x, y \mid A)=S_{0}(x, y) \mathcal{P} \exp -i g \int_{x^{0}}^{y^{0}} v_{\mu} A^{\mu} d t
$$

where $v_{\mu}$ is the 4-velocity of the quark, and $S_{0}(x, y)$ is the free propagator of the quark. Now, if one is looking at photons of very small invariant mass, the two quarks are nearly parallel in the collinear limit (even if the photon is hard), so that the two quarks have mostly the same $v$. As a consequence, the two path ordered exponentials cancel each other, and the product of the propagators under the functional integral is

$$
\gamma^{\mu} S(x, y \mid A) \gamma^{\nu} S(y, x \mid A) \approx \gamma^{\mu} S_{0}(x, y) \gamma^{\nu} S_{0}(y, x)
$$

\footnotetext{
${ }^{4}$ This action does not play any role in the argument, and therefore can even include the HTL effective action for gluons.
} 
Therefore, at the level of approximation at which the resummed quark propagator is calculated, the sum of all the gluon loop corrections is vanishing:

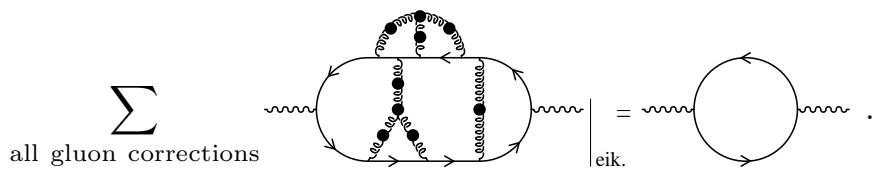

This result generalizes a result already known for QED established by 24, 25, 26, 27] for the subset of ladder vertex corrections, and extended to all abelian topologies in [28], to the case where gluons are exchanged (i.e. to non abelian topologies). Indeed, we see that what makes gluons specific, namely the fact that they can couple to each other, is hidden in the action $S[A]$, which plays a passive role in the argument.

This result is also closely related to the fact that there are no $\gamma \gamma g \cdots g$ HTL vertices (see [29] for an interpretation of the cancellation found in [24, 25, 28] as

a consequence of the absence of HTL vertices with $n>2$ photons), because the sum of all the eikonal contributions can also be written as (when $Q$ is soft):

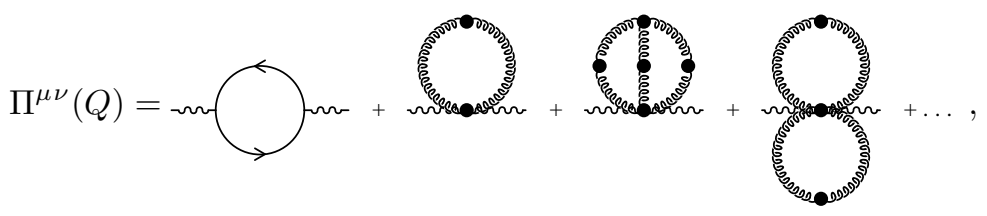

where the sum is extended to all the possible ways to close the external gluonic legs of the $\gamma \gamma g \cdots g$ vertices. If is then obvious to see that the sum reduces to its first term if these vertices are vanishing.

One is therefore left with the bare 1-loop diagram, which does not contributes to the imaginary part of the photon polarization tensor. In other words, the results derived in Eqs. (9) and (10) as a warmup and that were claimed to be related to the LPM effect in 21, are just artifacts with no physical meaning. In section 6 of the present paper, we show where the LPM effect appears in thermal field theory.

Despite the fact that this contribution is not physical, one learns two things from this calculation: (1) one has to keep terms beyond the eikonal approximation in order to circumvent this cancellation, and (2) the width may open the phase space to new processes.

\section{2-loop calculation}

The lesson from the previous section is that physical contributions must be looked for beyond the eikonal approximation. This implies inserting explicitly a gluon exchange with momentum $L$ in the diagram, and not assuming that $L^{2} \ll 2 P \cdot L$.

There are in principle two 2-loop topologies contributing to the photon polarization tensor. However, it was found in [16] that only the topology correcting 
the $q \bar{q} \gamma$ vertex (see Fig. 2) is relevant in the region where the collinear enhancement takes place. Since our purpose it to study how this collinear enhancement is affected by the width $\Gamma$, we limit the present study to the terms that were found important in 16$]$.

(b) (c)

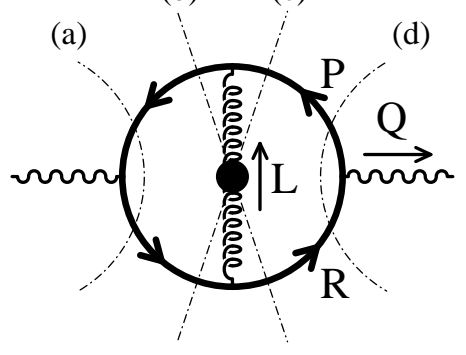

Figure 2: 2-loop vertex diagram. The dotted lines are the various cuts contributing to the imaginary part of the photon polarization tensor. The boldface quark line denotes the resummation of a width on the fermion propagator. The dot on the gluon indicates the resummation of HTLs on its propagator.

There is though one difference with the case $\Gamma=0$ that we must take into account: with a zero width, processes corresponding to cuts $(a)$ and $(d)$ are kinematically forbidden for small $Q^{2}$. As in the previous section, turning on the width opens those production channels, and we cannot disregard them a priori. This is not a problem though from a purely technical point of view, because it turns out that the sum of all the cuts has a simpler expression than each individual piece兯.

If we select only terms that have the large Bose-Einstein factor $n_{B}\left(l_{o}\right)$, the contribution of Fig. 2 to the imaginary part of the photon polarization tensor takes the following simple form 16

$$
\begin{aligned}
\operatorname{Im} \Pi_{\mu}^{\mu}(Q) \approx \frac{1}{2} e^{2} g^{2} \int \frac{d^{4} P}{(2 \pi)^{4}}\left[n_{F}\left(r_{0}\right)-n_{F}\left(p_{0}\right)\right] \\
\quad \times \int \frac{d^{4} L}{(2 \pi)^{4}} n_{B}\left(l_{0}\right) \rho_{T, L}(L) P_{T, L}^{\rho \sigma}(L) \operatorname{Tr}_{\rho \sigma} \\
\times\left[\boldsymbol{\Delta}_{R}(P) \boldsymbol{\Delta}_{R}(P+L)-\boldsymbol{\Delta}_{A}(P) \boldsymbol{\Delta}_{A}(P+L)\right] \\
\times\left[\boldsymbol{\Delta}_{R}(R) \boldsymbol{\Delta}_{R}(R+L)-\boldsymbol{\Delta}_{A}(R) \boldsymbol{\Delta}_{A}(R+L)\right],
\end{aligned}
$$

where $\rho_{T, L}(L)$ are the spectral functions of transverse and longitudinal gluons, $P_{T, L}^{\rho \sigma}(L)$ are the corresponding projectors, and where $\operatorname{Tr}_{\rho \sigma}$ is the result of the Dirac's trace for the quark loop. The result of the previous section requires that we keep in this Dirac's trace only terms that do not appear in the eikonal approximation (i.e. which includes soft corrections to the hard loop momentum). In

\footnotetext{
${ }^{5}$ In the appendix 4 A we show how one can obtain the difference between the cuts $(c)$ and (d). Having already their sum, we can therefore reconstruct the two contributions separately.
} 
the collinear limit, the first non vanishing term (beyond eikonal approximation) is

$$
\operatorname{Tr}_{\rho \sigma} \approx-8 L^{2}\left(R_{\rho} R_{\sigma}+P_{\rho} P_{\sigma}\right),
$$

which turns out to be the same as the term found in [16]. Its contractions with the projectors are given by

$$
\begin{aligned}
& P_{T}^{\rho \sigma}(L) \operatorname{Tr}_{\rho \sigma} \approx-8 L^{2}\left(r^{2}+p^{2}\right)\left(1-\cos ^{2} \theta^{\prime}\right) \\
& P_{L}^{\rho \sigma}(L) \operatorname{Tr}_{\rho \sigma} \approx+8 L^{2}\left(r^{2}+p^{2}\right)\left(1-\cos ^{2} \theta^{\prime}\right),
\end{aligned}
$$

where $\theta^{\prime}$ is the angle between the 3 -vectors $\boldsymbol{p}$ and $\boldsymbol{l}$. At this point, we have used the fact that we are looking at collinearly enhanced terms, and consider that $\boldsymbol{p}$ and $\boldsymbol{r}$ are parallel (the only place where we do not do this approximation is in the denominators which are very sensitive to the details of the collinear sector).

In order now to perform the integral over $p_{0}$, we follow the method of the previous section, and use the same approximations concerning the statistical weights. In addition, we compute only the contribution of cuts $(c)+(d)$, and multiply the result by an overall factor 2 in order to take into account the contribution of the cuts $(a)+(b)$. Following the remark of the previous section, we can start directly from the expression:

$$
\begin{aligned}
\operatorname{Im} \Pi_{\mu}^{\mu}(Q) \approx & \frac{1}{2} e^{2} g^{2} \int \frac{d^{4} P}{(2 \pi)^{4}}\left[n_{F}\left(r_{0}\right)-n_{F}\left(p_{0}\right)\right] \\
& \times \int \frac{d^{4} L}{(2 \pi)^{4}} n_{B}\left(l_{0}\right) \rho_{T, L}(L) P_{T, L}^{\rho \sigma}(L) \operatorname{Tr}_{\rho \sigma} \\
& \times 2 \pi \epsilon\left(p_{0}\right) \delta\left(P^{2}-M_{\infty}^{2}\right) \frac{1}{(P+L)^{2}-M_{\infty}^{2}} \\
& \times \operatorname{Disc}\left[\boldsymbol{\Delta}_{R}^{2 \Gamma}(R) \boldsymbol{\Delta}_{R}^{2 \Gamma}(R+L)\right],
\end{aligned}
$$

where we use the notation $\operatorname{Disc} f(\Gamma) \equiv f(\Gamma)-f(-\Gamma)$. We first do the $p_{0}$ integration for free thanks to the $\delta\left(P^{2}-M_{\infty}^{2}\right)$. In this section, we consider only the case of $p_{0}=+\omega_{p}$ (bremsstrahlung of a quark) in order to keep the calculation compact. The contribution of $p_{0}<0$ is identical if $q_{0} \ll T$, but is different if $q_{0}$ is large. In the latter case, the corresponding process is a $q^{*} \bar{q}$ annihilation $\left(q^{*}\right.$ denotes a quark placed off-shell by a scattering) instead of bremsstrahlung (see Fig. 3), and is considered in more detail in section 7 .

Then, it happens that the angular integral over the direction $\Omega_{l}$ of the 3vector $\boldsymbol{l}$ can be done analytically in a rather simple way. We have to perform an integral like

$$
I_{L} \equiv \int \frac{d \Omega_{l}}{4 \pi} \frac{1}{2 L \cdot P+L^{2}} \frac{1}{2 L \cdot R+L^{2}+2 P \cdot Q+Q^{2}+4 i r_{0} \Gamma},
$$

\footnotetext{
${ }^{6}$ Strictly speaking, we have also a factor of $1-\cos ^{2} \theta^{\prime}$ in the numerator that depends on $\Omega_{l}$. This factor can be taken into account analytically in the angular integral, the price to pay being more cumbersome expressions. One can however make the following simplification: if the width is small and if we use the collinear approximation for the numerators, then this angle is approximately given by $\cos \theta^{\prime} \approx l_{0} / l$, and does not play any role in the angular integrals.
} 

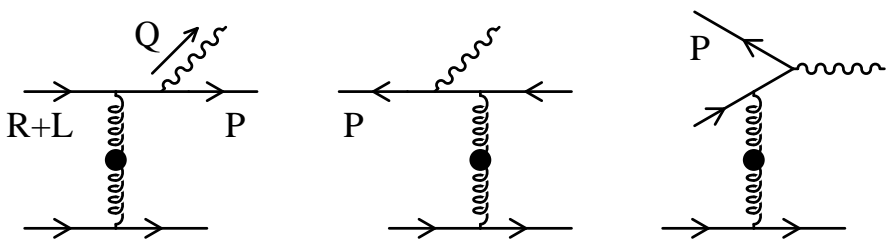

Figure 3: Different processes contained in the 2-loop diagram of Fig. 2. Left: $p_{0}=+\omega_{p}>0$. The corresponding process is the bremsstrahlung of a quark. Middle: $p_{0}=-\omega_{p}<0$ and $\omega_{p}>q_{0}$ so that $r_{0}=p_{0}+q_{0}$ (and also $\left.r_{0}+l_{0}\right)$ is negative as well. This region contains the bremsstrahlung of an antiquark. Right: $p_{0}=-\omega_{p}<0$ and $\omega_{p}<q_{0}$ so that $r_{0}=p_{0}+q_{0}$ (and also $r_{0}+l_{0}$ ) is positive. The corresponding process is the annihilation of an antiquark with an off-shell quark.

which can be rewritten as

$$
I_{L}=\int \frac{d \Omega_{l}}{4 \pi} \frac{1}{2 \widehat{L} \cdot A} \frac{1}{2 \widehat{L} \cdot B}
$$

where $\widehat{L} \equiv(1, \hat{\boldsymbol{l}})$ provided we introduce the fictitious " 4 -vectors"

$$
\begin{aligned}
& A \equiv\left(p_{0} l_{0}+\frac{L^{2}}{2}, l \boldsymbol{p}\right) \\
& B \equiv\left(r_{0} l_{0}+\frac{L^{2}}{2}+P \cdot Q+\frac{Q^{2}}{2}+2 i r_{0} \Gamma, l \boldsymbol{r}\right) .
\end{aligned}
$$

The advantage of rewriting $I_{L}$ like this lies in the fact that the last integral is known in closed form?:

$$
I_{L}=\frac{1}{8 \sqrt{\Delta}}[\ln (A \cdot B+\sqrt{\Delta})-\ln (A \cdot B-\sqrt{\Delta})],
$$

where $\Delta \equiv(A \cdot B)^{2}-A^{2} B^{2}$. We need now to evaluate the three quantities $A^{2}$, $B^{2}$ and $A \cdot B$, for which we will also use approximations based on the fact that $L$ is soft while the other momenta are hard. We obtain first a rather simple, and by now very familiar [16], expression for $\Delta$ :

$$
\Delta \approx p^{4} q_{0}^{2} l^{2}\left[\left(1-\cos \theta+\frac{M_{\mathrm{eff}}^{2}}{2 p^{2}}+\frac{L^{2}}{2 p^{2}}\right)^{2}-\frac{L^{2}}{p^{2}} \frac{M_{\mathrm{eff}}^{2}}{p^{2}}\right]
$$

where $\theta$ is the angle between the 3 -vectors $\boldsymbol{p}$ and $\boldsymbol{q}$, and with

$$
M_{\mathrm{eff}}^{2} \equiv M_{\infty}^{2}+\frac{Q^{2}}{q_{0}^{2}} p\left(p+q_{0}\right)+4 i \frac{\Gamma}{q_{0}} p\left(p+q_{0}\right) .
$$

\footnotetext{
${ }^{7} I_{L}$ is an analytic function of its arguments. The two possible choices for the square root of the complex number $\Delta$ lead to the same result.
} 
Therefore, apart from the fact that the effective mass $M_{\text {eff }}^{2}$ now gets an imaginary part coming from the width (and has been extended to hold for hard $q_{0}$ as well), this quantity is nothing but the denominator appearing in Eq. (39) of [16].

Since $A \cdot B \approx p r L^{2} \gg \sqrt{\Delta}$ and since the bremsstrahlung lives in the region where $L^{2}<0$, the difference of the two logarithms in Eq. (23) is just the discontinuity of the logarithm across its branch cut, which gives

$$
\ln (A \cdot B+\sqrt{\Delta})-\ln (A \cdot B-\sqrt{\Delta}) \approx 2 i \pi \epsilon(\Gamma) .
$$

Therefore, in presence of a width $\Gamma$, the contribution of bremsstrahlung to the photon polarization tensor is

$$
\begin{aligned}
\operatorname{Im}_{\mu}^{\mu} & (Q) \approx \frac{e^{2} g^{2}}{32 \pi^{4}} \frac{T}{q_{0}^{2}} \int_{0}^{+\infty} d p \frac{p^{2}+\left(p+q_{0}\right)^{2}}{p^{2}}\left[n_{F}\left(p+q_{0}\right)-n_{F}(p)\right] \\
\times & \int \frac{d l_{0}}{l_{0}} \int l^{3} d l\left[\rho_{T}\left(l_{0}, l\right)-\rho_{L}\left(l_{0}, l\right)\right]\left[1-\left(\frac{l_{0}}{l}\right)^{2}\right] \\
& \times \operatorname{Disc} \int_{0}^{2} \frac{\epsilon(\Gamma) d u}{\left[u+\frac{M_{\text {eff }}^{2}}{2 p^{2}}\right]\left[\left(u+\frac{M_{\text {eff }}^{2}}{2 p^{2}}+\frac{L^{2}}{2 p^{2}}\right)^{2}-\frac{L^{2}}{p^{2}} \frac{M_{\text {eff }}^{2}}{p^{2}}\right]^{1 / 2}},
\end{aligned}
$$

where $u \equiv 1-\cos \theta$. At this stage, this expression is formally similar to the one found for $\Gamma=0$ and reproduces known results in this limit. Indeed, when $\Gamma=0$, then $M_{\text {eff }}^{2}$ is a real number, and taking the discontinuity just gives a factor of 2 (do not forget the $\epsilon(\Gamma)$ in the numerator). It is then possible to use the very same sequence of changes of variables as in [16] to transform the integral over $u$, and write $\operatorname{Im}_{\mu}^{\mu}(Q)$ as

$$
\begin{aligned}
\operatorname{Im} \Pi^{\mu}{ }_{\mu}(Q) \approx \frac{2 e^{2} g^{2}}{\pi^{4}} & \frac{T}{q_{0}^{2}} \int_{0}^{+\infty} d p \frac{p^{2}+\left(p+q_{0}\right)^{2}}{2}\left[n_{F}\left(p+q_{0}\right)-n_{F}(p)\right] \\
& \times \sum_{m=T, L} \int_{0}^{1} \frac{d x}{x} \int_{0}^{+\infty} d w \frac{\left|\widetilde{I}_{m}\right| K(w, \widehat{\Gamma})}{\left(w+\widetilde{R}_{m}\right)^{2}+\left(\widetilde{I}_{m}\right)^{2}}
\end{aligned}
$$

where we denote:

$$
\begin{aligned}
& w \equiv \frac{-L^{2}}{\operatorname{Re} M_{\mathrm{eff}}^{2}}, \quad x \equiv \frac{l_{0}}{l}, \quad \widehat{\Gamma} \equiv \frac{\operatorname{Im} M_{\mathrm{eff}}^{2}}{\operatorname{Re} M_{\mathrm{eff}}^{2}} \\
& \widetilde{I}_{T, L} \equiv \frac{\operatorname{Im} \Pi_{T, L}^{H T L}}{\operatorname{Re} M_{\mathrm{eff}}^{2}}, \quad \widetilde{R}_{T, L} \equiv \frac{\operatorname{Re} \Pi_{T, L}^{H T L}}{\operatorname{Re} M_{\mathrm{eff}}^{2}},
\end{aligned}
$$

\footnotetext{
${ }^{8}$ We used the fact that the spectral functions $\rho_{T, L}$ have a simple expression in the space-like region:$$
\rho_{T, L}(L)=\frac{-2 \operatorname{Im} \Pi_{T, L}^{H T L}(L)}{\left(L^{2}-\operatorname{Re} \Pi_{T, L}^{H T L}(L)\right)^{2}+\left(\operatorname{Im} \Pi_{T, L}^{H T L}\right)^{2}} .
$$ 
and where the function $K$ comes from the integral over $u$ and is defined as 9

$$
\begin{aligned}
& K(w, \widehat{\Gamma}) \equiv \frac{1}{2} \int_{0}^{1} \frac{d y}{\sqrt{1-y}} \frac{y+4 / w}{(y+4 / w)^{2}+(4 \widehat{\Gamma} / w)^{2}} \\
&=\frac{1}{4\left(\alpha^{2}+\beta^{2}\right)}\left\{\alpha \ln \left(\frac{(1+\alpha)^{2}+\beta^{2}}{(1-\alpha)^{2}+\beta^{2}}\right)\right. \\
&\left.-2 \beta\left[\arctan \left(\frac{\alpha+1}{\beta}\right)-\arctan \left(\frac{\alpha-1}{\beta}\right)\right]\right\}
\end{aligned}
$$

with $\alpha+i \beta$ a square root $\sqrt{0}$ of the complex number $(4+w) / w+4 i \widehat{\Gamma} / w$. This function $K(w, \widehat{\Gamma})$ is the generalization to the case of a non vanishing width of the factor $\sqrt{w /(w+4)} \tanh ^{-1} \sqrt{w /(w+4)}$ appearing in Eq. (89) of [16]. In the limit of vanishing width $(\widehat{\Gamma} \rightarrow 0)$, we recover the results of $[16]$. Eq. $(29)$ cannot be further simplified analytically (except in some limiting cases), and must be evaluated numerically.

\section{Discussion}

\subsection{Modification of the emission spectrum}

By inspecting our final expression given in Eq. (29), the first thing we notice is the very strong similarity with the same result in the absence of the width. Only the function $K(w, \widehat{\Gamma})$ contains the width, in the form of the dimensionless ratio $\widehat{\Gamma}$. When this ratio is small, the width has a negligeable effect while on the contrary if $\widehat{\Gamma} \gg 1$ then the width plays a dominant role.

A first simple conclusion is obtained by noticing that the width arrives in $M_{\text {eff }}^{2}$ via the combination $\Gamma p\left(p+q_{0}\right) / q_{0}$ which becomes large at small $q_{0}$. Therefore, the effect of the width is more important at small $q_{0}$. This property is illustrated by the plot of Fig. 1 , which shows $\operatorname{Im} \Pi^{\mu}{ }_{\mu}$ (obtained numerically from Eq. (29)) as a function of the photon energy $q_{0}$ (the invariant mass $Q^{2}$ is kept zero in this plot), for different values of the width $\Gamma$. On this plot, one can see that the $q_{0}^{-1}$ behavior of bremsstrahlung at small $q_{0}$ is modified in presence of a width. Instead of that, one reaches a plateav 10 when $q_{0} \rightarrow 0$. By

${ }^{9}$ One can go from the variable $u$ to $y$ by the following transformations

$$
2 p^{2} u \equiv M_{\text {eff }}^{2}\left[w(1-z)+z^{-1}-1\right], \quad \text { and } y \equiv 4\left(z-z^{2}\right) .
$$

${ }^{10}$ Explicitly, we have:

$$
\alpha, \beta=\sqrt{\frac{1}{2}\left[\sqrt{((4+w) / w)^{2}+(4 \widehat{\Gamma} / w)^{2}} \pm((4+w) / w)\right]} .
$$

${ }^{11} \mathrm{~A}$ similar behavior has already been observed in a very different calculation by Weldon in [30] (see Fig. 1 of [30]). However, the resummations considered in this paper deal with the possibility to emit more than one photon, and affect the spectrum only below the scale 


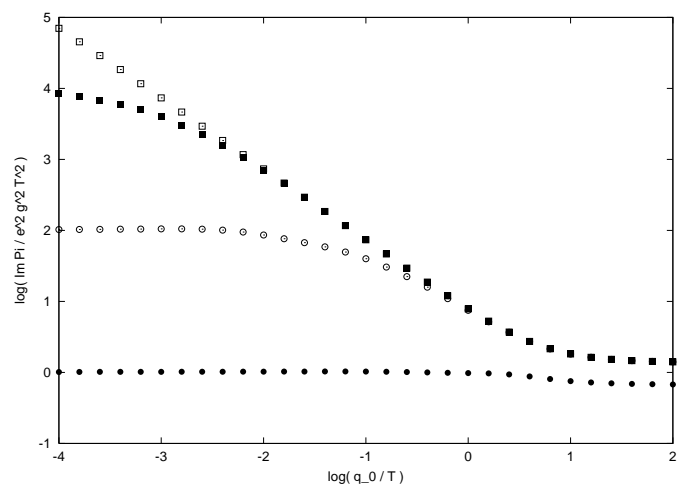

Figure 4: Effect of the width on the bremsstrahlung as a function of $q_{0} / T$ (for $\left.Q^{2}=0\right)$. The various curves correspond to different values of the width $\Gamma$. From top to bottom, the ratio $\Gamma T / M_{\infty}^{2}$ (which corresponds to $\widehat{\Gamma} / 4$ when $q_{0} \gg T$ ) takes the values $10^{-6}, 10^{-4}, 10^{-2}$ and 1 .

comparing the second and third curves, we see that the value of $q_{0}$ at which we reach this plateau varies by a factor $10^{2}$ when $\Gamma$ varies by the same factor. This is a consequence of the fact that the width enters in the result via the ratio $\Gamma / q_{0}$ for small $q_{0}$. Moreover, the value of the plateau is proportional to $1 / \Gamma$ (this can be deduced from the plot since $\Gamma$ increases by a factor $10^{2}$ between two consecutive curves). This leads to the conjecture that the rate has a very simple $\Gamma$ dependence in the region where the width is dominant. Additionally, when $\Gamma$ increases, the extension of this plateau increases as well. In particular, for a large enough width, even the region of hard photons is modified.

\subsection{Region where the width is important}

If one wants to go beyond this qualitative statement, and get a better idea of the region (in the $\left(q, q_{0}\right)$ plane) where the width is important, one must study the ratio $\widehat{\Gamma}$. Indeed, the curve defined by the equation $\widehat{\Gamma}=1$ is precisely the curve on which the effect of the width is comparable to the effect of $M_{\infty}^{2}$ and

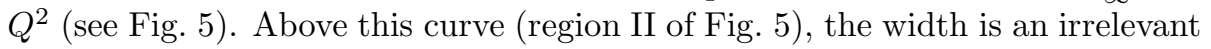
parameter, and below this curve (region I - low invariant mass photons) we have the region where the width is the dominant collinear regulator.

At first sight, the region where the width is important looks very similar to the region where higher loop corrections are IR-sensitive to the scale $g^{2} T$ and must be resummed, as found in [18]. We recall that in that work we considered the infrared structure of higher loop diagrams contributing to thermal photon production. It was shown that compensations between different cuts cancel

$q_{0} \sim \alpha_{E M} T$, much smaller than the scale at which $\Gamma \sim g^{2} T \ln (1 / g)$ starts playing a role. The effect we are considering here appears at much larger photon energies (even including hard photons if $\Gamma$ is large enough), since it is related to resummations of gluons and thus involves the strong coupling constant $\alpha_{S} \gg \alpha_{E M}$. 


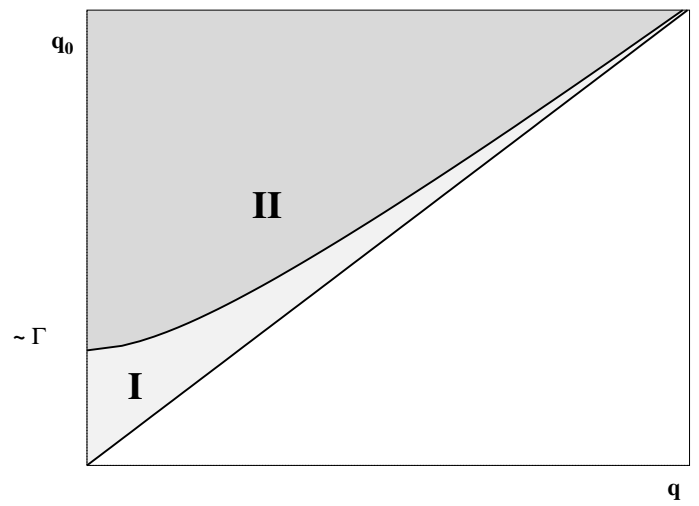

Figure 5: Boundary obtained from the condition $\widehat{\Gamma}=1$. In region $I$, the width is the dominant regulator of collinear singularities. In addition, the shape of the emitted spectrum is modified by the width. In region II, the width is only a sub-dominant correction.

all unscreened infrared divergences, and that the remaining terms are sensitive to gluon momenta down to a $Q$-dependent cutoff. In addition, we compared this cutoff with the scale $\mu \sim g^{2} T$ of the magnetic mass, and concluded that higher loop diagrams would be sensitive to the magnetic mass for a small enough photon invariant mass (see the figure 5 of $[18]$ ).

This similarity comes from the fact that line in the $\left(q, q_{0}\right)$ plane where $\Gamma$ starts to be important is given by the equation:

$$
2 \Gamma \sim \frac{q_{0}}{2} \frac{\operatorname{Re} M_{\mathrm{eff}}^{2}}{p\left(p+q_{0}\right)} .
$$

while the line on which the magnetic mass $\mu$ becomes important has the following equation:

$$
\mu \sim \frac{q_{0}}{2} \frac{\operatorname{Re} M_{\mathrm{eff}}^{2}}{p\left(p+q_{0}\right)} .
$$

In other words, the two lines are defined by comparing a common momentum scale alternatively with the width of the quarks and with the magnetic mass. The physical interpretation of the momentum scale appearing in the right hand side of Eqs. (34) and (35) will be given in the next section. The two boundaries are therefore very similar in QCD because $\Gamma \sim g^{2} T \ln (1 / g)$ and $\mu \sim g^{2} T$ are not very different. But on the other hand, $\Gamma$ and $\mu$ have very different meanings $\sqrt{12}$, and we may expect rather different interpretations for the two conditions Eqs. (34) and (35). In the next section, we show that Eq. (34) is closely related to the LPM effect.

There is also a more technical argument suggesting the difference of Eqs. (34) and (35), which becomes clear by examining how the width $\Gamma$ would appear in

\footnotetext{
${ }^{12}$ In particular, the fact that $\Gamma$ and $\mu$ are very close is specific to QCD. In QED, one would have $\mu=0$ and $\Gamma \sim e^{2} T \ln (1 / e)$.
} 
perturbation theory. For that purpose, let us insert a self-energy correction on the leg of momentum $R$ in the two-loop diagram of Fig. 2. Since we are dealing with the width perturbatively here, the quark propagators contain only the asymptotic mass $M_{\infty}$. By a crude power counting, we can estimate that this insertion brings the following extra factor ${ }^{13}$

$$
\frac{i r^{0} \Gamma}{R^{2}-M_{\infty}^{2}} \sim \frac{1}{\operatorname{Re} M_{\mathrm{eff}}^{2}} \frac{i \Gamma p\left(p+q_{0}\right)}{q_{0}} .
$$

We can already see that a self-energy insertion increases the strength of the potential collinear divergences by bringing an additional denominator $R^{2}-M_{\infty}^{2}$. On the other hand, this insertion does not modify the infrared properties of this diagram. By summing over the number of such insertions from 0 to $+\infty$, we get a factor

$$
\frac{\operatorname{Re} M_{\text {eff }}^{2}}{\operatorname{Re} M_{\text {eff }}^{2} \oplus i \Gamma p\left(p+q_{0}\right) / q_{0}} .
$$

Therefore, the effect of such a resummation is to substitute $\operatorname{Re} M_{\text {eff }}^{2}$ by $\operatorname{Re} M_{\text {eff }}^{2} \oplus$ $i \Gamma p\left(p+q_{0}\right) / q_{0}$ in the factor $T^{2} / M_{\text {eff }}^{2}$ of collinear enhancement. This is precisely what has been observed in the more rigorous calculation of section 4 . In addition, this simple argument demonstrates clearly that the mode of action of width insertions is to affect the collinear sector, leaving unmodified the infrared sector. On the contrary, the possibility to have a sensitivity to $\mu$ found in [18] is related to infrared singularities due exclusively to transverse gluonst

\subsection{Limit of dominant width}

In region I, where the width becomes the dominant regulator, the ratio $\widehat{\Gamma}$ is large, which enables us to make some additional approximations. In particular, we can perform very simply the integrals over $w$ and $y$ in Eq. (29). Indeed, we can first note that $\widehat{\Gamma}$ sets the order of magnitude of the variable $w$. As a consequence, typical values of $w$ are large, and we can neglect the corrections $\widetilde{R}_{T, L}$ and $\widetilde{I}_{T, L}$ in the denominator of Eq. (29), as well as $1 / w$ in front of $y$. Therefore, this equation becomes

$$
\begin{aligned}
\operatorname{Im} \Pi_{\mu}^{\mu}(Q) & \approx \frac{2 e^{2} g^{2}}{\pi^{4}} \frac{T}{q_{0}^{2}} \int_{0}^{+\infty} d p \frac{p^{2}+\left(p+q_{0}\right)^{2}}{2}\left[n_{F}\left(p+q_{0}\right)-n_{F}(p)\right] \\
\times & {\left[\sum_{m=T, L} \int_{0}^{1} \frac{d x}{x}\left|\widetilde{I}_{m}\right|\right]\left[\int_{0}^{+\infty} \frac{d w}{w^{2}} \frac{1}{2} \int_{0}^{1} \frac{d y}{\sqrt{1-y}} \frac{y}{y^{2}+(4 \widehat{\Gamma} / w)^{2}}\right] }
\end{aligned}
$$

\footnotetext{
${ }^{13}$ A self-energy correction of the type considered here modifies the real-part of the pole of the propagator by a mass-shift $\delta M_{\infty}^{2}$, negligible compared to $M_{\infty}^{2}$ arising from HTL corrections.

${ }^{14}$ In particular, longitudinal as well as transverse gluons contribute to $\Gamma$. There is no contradiction with [18] which found only the transverse gluons to be important, since the statement of 18 was about infrared singularities.
} 


$$
=\frac{2 e^{2} g^{2}}{\pi^{4}} \frac{T}{q_{0}^{2}} \int_{0}^{+\infty} d p \frac{p^{2}+\left(p+q_{0}\right)^{2}}{2}\left[n_{F}\left(p+q_{0}\right)-n_{F}(p)\right] \times \frac{3 \pi m_{\mathrm{g}}^{2}}{2 \operatorname{Re} M_{\mathrm{eff}}^{2}} \frac{\pi}{4 \widehat{\Gamma}}
$$

where $m_{\mathrm{g}} \sim g T$ is the gluon thermal mass coming from the prefactor of $\widetilde{I}_{T, L}$. We see that this expression is of the form

$$
\operatorname{Im} \Pi_{\mu}^{\mu}(Q) \approx e^{2} g^{4} \frac{T^{3}}{\Gamma} \Phi\left(\frac{q_{0}}{T}\right),
$$

where $\Phi$ is a function independent of $\Gamma$ giving the shape of the photon spectrum. Therefore, in the region where the width dominates, the spectrum scales as $\Gamma^{-1}$. One notes that the combination $\widehat{\Gamma} \operatorname{Re} M_{\text {eff }}^{2}$ is proportional to $\Gamma p\left(p+q_{0}\right) / q_{0}$, and therefore the shape of the spectrum is independent of $Q^{2}$ and $M_{\infty}^{2}$. Furthermore, for $q_{0} \ll T$, it is independent of $q_{0}$ (see Fig. (4).

It may be interesting to compare in two extreme cases $\left(q_{0}\right.$ soft and $\left.q_{0} \gg T\right)$ the result of Eq. (39) with those obtained with a vanishing width. In the soft $q_{0}$ case, the result when $\Gamma=0$ was obtained in 16]:

$$
\operatorname{Im} \Pi_{\mu}^{\mu}(Q) \sim e^{2} g^{4} \frac{T^{3}}{q_{0}} \frac{1}{g^{2}},
$$

where we have explicitly isolated the factor $T^{2} / M_{\infty}^{2} \sim 1 / g^{2}$ that comes from the collinear enhancement, while we have now:

$$
\operatorname{Im} \Pi^{\mu}{ }_{\mu}(Q) \sim e^{2} g^{4} \frac{T^{3}}{q_{0}} \frac{q_{0}}{\Gamma} .
$$

The factor $q_{0} / \Gamma$ is in fact the new enhancement factor coming from $T^{2} / \operatorname{Im} M_{\text {eff }}^{2}$. Therefore, the two results differ only by the nature of the factor of collinear enhancement. For hard photon bremsstrahlung, we had on the other hand [17]

$$
\operatorname{Im} \Pi_{\mu}^{\mu}(Q) \sim e^{2} g^{4} T^{2} \frac{1}{g^{2}}
$$

which becomes now

$$
\operatorname{Im} \Pi_{\mu}^{\mu}(Q) \sim e^{2} g^{4} T^{2} \frac{T}{\Gamma} .
$$

Again, the two expressions differ only by the collinear enhancement factor: $T^{2} / M_{\infty}^{2} \sim 1 / g^{2}$ if $\Gamma=0$ instead of $T^{2} / \operatorname{Im} M_{\text {eff }}^{2} \sim T / \Gamma$ when the width is dominant. The numerical prefactors not written in Eqs. (42) and (43) could however be quite different since they reflect a different physics.

The $\Gamma^{-1}$ scaling law in Eq. (39) has also a very simple physical interpretation related to the fact that $\Gamma^{-1}$ is the mean free path of the quark in the medium. This result just tells us that the photon rate is proportional to the mean free path of the quark that emits the photon. In other words, quarks colliding very frequently do not have enough time to emit photons. This picture is at the basis of the Landau-Pomeranchuk-Migdal effect, that we discuss in the next section. 


\section{Connection with the LPM effect}

\subsection{Generalities on the LPM effect}

The non-perturbative region found in the present paper can also be interpreted in a much more physical way, which seems to indicate that the region I in Fig. 5 is the region where the Landau-Pomeranchuk-Migdal [31, 32, 33, 34, 35] effect modifies photon production by a plasma.

Let us first recall the condition for the LPM effect, by using a very heuristic argument valid for real photon production. In the bremsstrahlung process of photon production, the gluon exchanged during the scattering has a minimal momentum given by 33.

$$
l_{\text {min }} \approx \frac{q_{0}}{2} \frac{M_{\infty}^{2}}{p\left(p+q_{0}\right)},
$$

where $p$ is the momentum of the quark. The inverse of this momentum transfer defines the "length" on which the photon is emitted, and for this reason is called the "coherence length" (denoted $\lambda_{\text {coh }}$ in the following) for the production of this photon. It is also interpreted as the "formation time" of the photon. If $\lambda_{\text {coh }}$ is much smaller that the typical distance between two consecutive scatterings (proportional to $1 / \Gamma$ ), then the photon rate is not affected by multiple scatterings. In other words, successive scatterings can be considered as independent. On the contrary, if the coherence length is larger than the mean free path, then successive scatterings are not independent anymore. This is the LPM effect. It is also possible to recast the previous condition as a comparison between the "formation time" and the mean free path of the quark in a very suggestive way: the photon must be produced before the quark scatters off another parton.

\subsection{LPM effect in thermal field theory}

Let us now show that the previous discussion arises automatically in the thermal field theory approach. In fact, there is in the calculation presented before a quantity very similar to the coherence length discussed in the semi-classical treatment of the LPM effect, namely,

$$
\lambda_{\mathrm{coh}}^{-1} \sim \frac{q_{0}}{2} \frac{\operatorname{Re} M_{\mathrm{eff}}^{2}}{p\left(p+q_{0}\right)} .
$$

This value agrees with the one of [31, 32, 33] when $Q^{2}=0$ since it comes from kinematics, and generalizes it to the case where the invariant mass $Q^{2}$ is non vanishing. Additionally, this quantity was found in 18 to be the lower bound for the momentum of exchanged gluons.

It is now possible to reformulate the condition $\widehat{\Gamma}=1$ in more physical terms. Indeed, this condition can be rewritten as

$$
\lambda_{\text {mean }} \sim \Gamma^{-1} \sim \lambda_{\text {coh }},
$$

where $\lambda_{\text {mean }}$ is the mean free path of the quark in the plasma, and the region where the width dominates corresponds to the condition $\lambda_{\text {mean }}<\lambda_{\text {coh }}$. At this 
point, the connection with the LPM effect is obvious: the region we found to be non-perturbative due to the width of the quarks is also the region where the LPM effect matters.

This discussion on the condition for the LPM effect to occur is in fact summarized in the expression for $M_{\text {eff }}^{2}$ introduced in Eq. (25). This formula can be written very elegantly as:

$$
M_{\text {eff }}^{2}=\frac{2 p\left(p+q_{0}\right)}{q_{0}}\left[\frac{1}{\lambda_{\text {coh }}}+\frac{i}{\lambda_{\text {mean }}}\right],
$$

and the physical condition for the LPM effect ${ }^{15}$ to be relevant is mathematically expressed by the dominance of $\operatorname{Im} M_{\text {eff }}^{2}$ over $\operatorname{Re} M_{\text {eff }}^{2}$. Conversely, when $\operatorname{Re} M_{\text {eff }}^{2}>\operatorname{Im} M_{\text {eff }}^{2}$, the perturbative approach is valid. As a side remark, let us note that the quantity $M_{\text {eff }}^{2}$, which controls the physics of bremsstrahlung, combines in a very simple way three soft scales of the problem: $Q^{2}, M_{\infty}^{2}$ and $\Gamma$. It is also possible to give a very simple physical interpretation of the ratio $\widehat{\Gamma}$ which contains all the $\Gamma$ dependence of the final result (Eq. (29)). Indeed, one can rewrite this ratio as $\widehat{\Gamma}=\lambda_{\text {coh }} / \lambda_{\text {mean }}$, which is nothing but the typical number of coherent scatterings necessary to produce a photon.

The analogy with the standard treatment of the LPM effect is only partial though. Indeed, our estimate of the effect of the finite mean free path of the quarks is too crude (because of the constant width, and because of the missing vertex corrections) to be reliable from a quantitative perspective. The trend found here (suppression of the photon spectrum at small $q_{0}$ ) is however expected to be a solid prediction, and is in agreement with what is usually found from the LPM effect.

The main difference with the usual calculation of the LPM effect comes from the treatment of scattering centers: one usually assumes a fast moving charged particle going through a medium of "cold" (static) scattering centers. In the case we are considering here, both the particle emitting the photon and the scattering centers are thermalized particles, of comparable momenta. The approximations made in [31, 32, 33, 34, 35] do not apply to this situation, and it is not clear whether a quantitative agreement is to be expected at all. In particular, the static approximation selects only Debye shielded longitudinal gluon exchanges, while the width (the resummation of which introduces multiple scatterings in our approach) of the thermalized quark receives contributions from transverse gluons as well. Contrary to [31, 32, 33, 34, 35], transverse gluon exchanges are very important in thermal field theory, and matter for the LPM effect as well.

There is a major difference between the LPM effect from multiple longitudinal gluon exchanges and multiple transverse gluon exchanges, which can be

\footnotetext{
${ }^{15}$ In [36] was investigated the influence of the finite mean free path of nucleons on axion (or neutrino pair) production by a supernova. The connection between the width $\Gamma$ and the LPM effect is also mentioned in this paper. However, the condition given by Eq. 46) does not come out from the formalism of [36] (it seems that the approximations made in 36 for the bremsstrahlung do not enable one to track the coherence length, so that only $\Gamma$ appears in the final result). What we have shown in the present section is that a careful calculation of the bremsstrahlung in thermal field theory generates automatically both terms of the comparison, through the effective mass given in Eq. (47).
} 
readily seen when one compares the respective ranges of electric and magnetic fields to the mean free path of the quark. Indeed, since $\lambda_{\text {mean }} \gg m_{D}^{-1} \sim(g T)^{-1}$, the mean free path is much larger than the range of Debye screened electric fields. As a consequence, successive exchanges of longitudinal gluons are independent: they correspond to scatterings off different partons. On the contrary, we have $\mu^{-1}>\lambda_{\text {mean }}$, which implies that the range of magnetic fields can extend beyond the mean free path of the quark. Therefore, successive exchanges of transverse gluons may correspond to scatterings off the same parton. This interpretation is supported by the infrared study performed in [18], where we found that only transverse gluons are causing trouble in the infrared sector, and that this problem occurs when $\lambda_{\text {coh }}>\mu^{-1}$ (see Eq. (35)). This condition means that the production of a single photon occurs on a distance larger than the correlation length of magnetic fields: this emission process is therefore able to probe the scale of the magnetic screening, and the rate is expected to become non perturbative. This qualitative difference of transverse gluon exchanges is also supported by the fact that the contribution of longitudinal gluons to $\Gamma$ is perturbative (saturated at 1-loop), while the transverse contribution to $\Gamma$ is non-perturbative [22, 23].

\subsection{Comparison with the approach of Cleymans et al.}

The LPM effect has already been studied in the context of photon production by a quark-gluon plasma, although with a very different approach, in [37]. In this paper, the study follows the semi-classical approach of [33], to first deduce the photon rate from a single quark trajectory, and then average over the possible trajectories. Finally, the authors of [37] manage to rewrite the rate as a function of a quantity $F(\boldsymbol{\theta}, \tau)$ which is related to the probability for the quark trajectory to undergo an angular deviation $\boldsymbol{\theta}$ after a time $\tau$. Additionally, they show that this object satisfies the following Fokker-Planck equation, which would be in our notations 10 :

$$
\frac{\partial F}{\partial \tau}+i \frac{q_{0}}{2}\left[\boldsymbol{\theta}^{2}+\frac{M_{\infty}^{2}}{p^{2}}+\frac{Q^{2}}{q_{0}^{2}}\right] F=\frac{\left\langle\boldsymbol{\theta}_{S}^{2}\right\rangle}{4} \nabla_{\theta}^{2} F,
$$

where $\left\langle\boldsymbol{\theta}_{S}^{2}\right\rangle$ is the mean square of scattering angle per unit length.

In order to make the connection with our approach more transparent, we can factorize out the quark width in the following way (recalling the fact that $\Gamma^{-1}$ gives the mean free path):

$$
\left\langle\boldsymbol{\theta}_{S}^{2}\right\rangle \equiv 16 \overline{\boldsymbol{\theta}^{2}} \Gamma
$$

where $\overline{\boldsymbol{\theta}^{2}}$ is the average square of the scattering angle per collision (up to a purely numerical factor) and where the prefactor 16 has been chosen for later

\footnotetext{
${ }^{16}$ In 37], only soft photons are considered. As a consequence, in this comparison, we assume always $q_{0} \ll p$, and therefore do not distinguish $p$ and $p+q_{0}$.
} 
convenience. We can now rewrite the equation satisfied by $F$ in the following way

$$
\frac{\partial F}{\partial \tau}+i q_{0}\left[\frac{\boldsymbol{\theta}^{2}}{2}+\frac{1}{2 p^{2}}\left\{\operatorname{Re} M_{\text {eff }}^{2}+i \operatorname{Im} M_{\text {eff }}^{2} \overline{\boldsymbol{\theta}^{2}} \nabla_{\theta}^{2}\right\}\right] F=0 .
$$

We see that this equation is governed by a complex number whose real and imaginary part also appear in our approach. In particular, the condition for having LPM effect (discussed after Eq. (2.21) of [37]) is the same as ours, given the fact that typical scattering angles satisfy $\overline{\boldsymbol{\theta}^{2}} \sim \operatorname{Re} M_{\text {eff }}^{2} / p^{2}$. Note however that our calculation also includes the case of hard photon production, and shows that transverse gluon exchanges are equally important, both points which are not contained in Cleymans et al.'s approach.

\section{$7 \quad q^{*} \bar{q}$ annihilation}

\subsection{Technical differences}

In section 1 , when we performed the integral over the quark energy $p_{0}$ using the $\delta\left(P^{2}-M_{\infty}^{2}\right)$ in Eq. 19, we considered only the positive energy solution, which corresponds to photon emission by the bremsstrahlung of a quark. The calculation is very similar for the contribution of $p_{0}=-\omega_{\boldsymbol{p}}$, with some peculiarities that we are going to highlight in this section.

One of the most important differences is that, since we have $q_{0}>0$, this contribution contains in fact the sum of two processes (see Fig. 3): the bremsstrahlung of an antiquark if $\omega_{p}>q_{0}$, and the annihilation of an off-shell quark (a quark put off-shell by a scattering) with an antiquark if $\omega_{p}<q_{0}$. The latter process has been studied in the case of zero width and real photons in [17] and was indeed found to be a very important contribution to hard photon production. The purpose of this section is not to present a complete calculation of this process (which would require the discussion of a few other technical issues, especially in the region where $Q^{2}>4 M_{\infty}^{2}$ ), but only to reproduce in this case the discussion of section 5, in order to have a qualitative picture of the influence of the width on this process.

The calculation of the $p_{0}<0$ contribution can be performed by the same method as the $p_{0}>0$ one. In particular, the angular integral over $d \Omega_{l}$ generates an effective mass $M_{\text {eff }}^{\prime 2}$, the expression of which is

$$
M_{\mathrm{eff}}^{\prime 2} \equiv M_{\infty}^{2}+\frac{Q^{2}}{q_{0}^{2}} p\left(p-q_{0}\right)+4 i \frac{\Gamma}{q_{0}} p\left(p-q_{0}\right) .
$$

In addition to this change, the angular integral over $d \Omega_{p}$ is now dominated by values of $\theta$ around $\pi$ (because $1-\cos \theta$ in the expression of $\Delta$ becomes $1+\cos \theta$ ) 17 . A practical consequence of this is that $r \approx\left|p-q_{0}\right|$ in the collinear limit. All

\footnotetext{
${ }^{17}$ This difference is also obvious when one compares the process on the left of Fig. 3 with the two processes on the right of the same figure, which we are dealing with now.
} 
the subsequent steps can be reproduced and one is lead to the following final formula:

$$
\begin{aligned}
\operatorname{Im} \Pi_{\mu}^{\mu}(Q) \approx \frac{2 e^{2} g^{2}}{\pi^{4}} & \frac{T}{q_{0}^{2}} \int_{0}^{+\infty} d p \frac{p^{2}+\left(q_{0}-p\right)^{2}}{2}\left[n_{F}\left(q_{0}-p\right)-n_{F}(-p)\right] \\
& \times \sum_{m=T, L} \int_{0}^{1} \frac{d x}{x} \int_{0}^{+\infty} d w \frac{\left|\widetilde{I}_{m}\right| K(w, \widehat{\Gamma})}{\left(w+\widetilde{R}_{m}\right)^{2}+\left(\widetilde{I}_{m}\right)^{2}},
\end{aligned}
$$

where the notations are the same as in Eq. (30), with $M_{\mathrm{eff}}^{\prime 2}$ in place of $M_{\mathrm{eff}}^{2}$.

Another very important technical difference appears when one performs the integral over the quark momentum $p$. Indeed, the range of this integral is controlled by the statistical factors $n_{F}\left(r_{0}\right)-n_{F}\left(p_{0}\right) \approx n_{F}(p)-n_{F}\left(p-q_{0}\right)$. This factor is of order 1 in a domain going from $p=0$ to $p \sim \operatorname{Max}\left(q_{0}, T\right)$. In other words, if $q_{0}$ is soft, this range is the usual $[0, T]$ interval. On the contrary, if $q_{0} \gg T$, the integral extends to $p \sim q_{0} \gg T$. A practical consequence is that for $q_{0} \ll T$, the contribution of $p_{0}<0$ comes mainly from the bremsstrahlung of an antiquark (since most of $d p$ integral satisfies $q_{0}<p \sim T$ ), while for $q_{o} \gg T$ it is dominated by the $q^{*} \bar{q}$ annihilation (since we then have $p<q_{0}$ ). In the intermediate region $q_{0} \sim T$, this contribution is a mixture of both processes.

\subsection{Modifications due to the width}

Again, we see that the term depending on $\Gamma$ in $M_{\text {eff }}^{\prime 2}$ comes with $\Gamma / q_{0}$, indicating that the width will modify significantly the rate when $q_{0}$ is soft. But, in addition, when $q_{0} \gg T, p$ can itself be of order $q_{0}$, so that the imaginary part of $M_{\mathrm{eff}}^{\prime 2}$ is of order $\Gamma q_{0}$, which can be much larger than $M_{\infty}^{2}$ at very large $q_{0}$. It means that the width also changes dramatically the spectrum of very hard real photons (in the case of bremsstrahlung, the width modifies only marginally the rate of hard photons, since the imaginary part of $M_{\mathrm{eff}}^{2}$ is of the same order of magnitude as $M_{\infty}^{2}$ if $q_{0} \gg T$ and $\Gamma \sim g^{2} T$ ) coming from the $q^{*} \bar{q}$ annihilation.

This has been checked by evaluating numerically Eq. (52), and the results are displayed in Fig. 6, where we plot the imaginary part of the photon polarization tensor as a function of $q_{0} / T$ (with the same values of temperature and coupling constant as in Fig. (1) for a set of values of $\Gamma$ identical to the one chosen in Fig. F. From this plot, it is obvious that the regions $q_{0} \ll T$ and $q_{0} \gg T$ are very different. In fact, in the region $q_{0} \ll T$, we obtain results that exactly match those of Fig. 4 indeed, in this region, the $p_{0}<0$ contribution is dominated by the bremsstrahlung of an antiquark, which as expected contributes equally as the bremsstrahlung of a quark.

The new feature coming with $p_{0}<0$ appears in the hard photon region $\left(q_{0} \gg T\right)$, where now the $q^{*} \bar{q}$ annihilation contributes. At very small $\Gamma$ (upper curve), we recover the result of [17] according to which the process $q^{*} \bar{q} \rightarrow \gamma$ gives a contribution to $\operatorname{Im} \Pi^{\mu}{ }_{\mu}(Q)$ that increases like $q_{0}$. Then, as $\Gamma$ increases, we see a saturation at large enough $q_{0}$, to a value that behaves like $\Gamma^{-1}$. This 


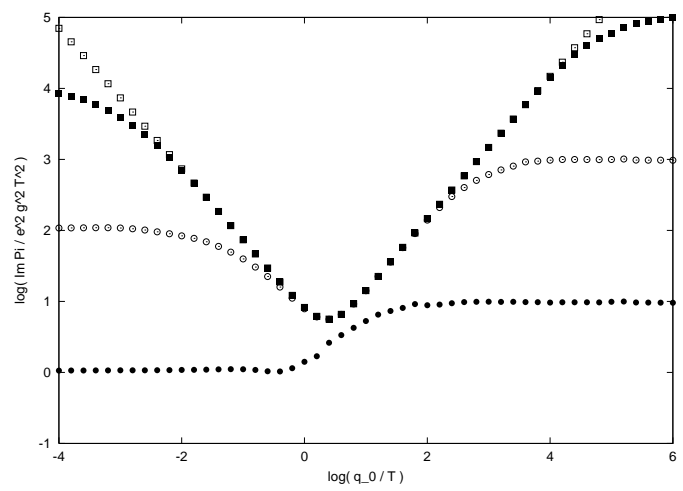

Figure 6: Effect of the width in the region $p_{0}<0$ as a function of $q_{0} / T$ (for $\left.Q^{2}=0\right)$. Each curve corresponds to a different value of the width $\Gamma$. The set of values taken for $\Gamma$ is the same as in Fig. 6 .

confirms the above qualitative statement based on $M_{\mathrm{eff}}^{\prime 2}$. In this case also, it is possible to obtain a formula like Eq. (39) showing explicitly the $\Gamma$ scaling law when $\Gamma$ is the dominant collinear regulator.

The contribution of the process $q^{*} \bar{q} \rightarrow \gamma$, first evaluated at leading order in [17], has been found to be phenomenologically important for the production of direct photons in the $2-10 \mathrm{GeV}$ range, because it dominates previous estimates by a factor of order 5 [38]. The inclusion of this contribution in heavy nuclei collisions simulations [39] has lead to a good agreement with the measured rates from the WA98 experiment 40, 41]. These rates did not include the LPM suppression advocated here, but one must realize that the energy range where thermal photons are relevant is also the region of minimum sensitivity to the width $\Gamma$ (around the minimum of the curves in figure 6), since for a temperature of a few hundred $\mathrm{MeV}$, the $\mathrm{GeV}$ range corresponds to $q_{0} / T \sim 10$. It is too early to be more quantitative here given the fact that vertex corrections 42 have been completely disregarded in the present work, but having a precise prediction for this process would definitely be of important phenomenological interest.

\subsection{LPM effect in $q^{*} \bar{q}$ annihilation}

When $p_{0}<0$, the condition under which the width is the dominant collinear regulator can be rewritten as

$$
2 \Gamma>\frac{q_{0}}{2} \frac{\operatorname{Re} M_{\mathrm{eff}}^{\prime 2}}{p\left|q_{0}-p\right|},
$$

and the quantity in the right hand side is the minimal value $l_{\min }$ for the momentum $L$ of the gluon exchanged in the scattering. Therefore, its inverse is also the coherence length for the emission process, and the above condition is nothing but the criterion for having LPM effect. 
As a consequence, we are lead to the conclusion that the LPM effect plays a role not only for the photon production by bremsstrahlung at low energy, but also for the $q^{*} \bar{q}$ annihilation at high energy. This conclusion was also recently obtained in [43 by a semi-classical method following the original method of Migdal [33]. The authors of [43] in fact studied the inverse of the process $q^{*} \bar{q} \rightarrow \gamma$, namely the production of a fermion pair out of a photon (this process is made possible by the fact that one of the fermions is produced off-shell and then scatters in the medium). They found that the LPM effect is important for the fragmentation of very high energy photons. This is equivalent to our observation that the LPM effect modifies $q^{*} \bar{q} \rightarrow \gamma$ for large $q_{0}$.

\section{Conclusions}

In this paper, we have studied the effect of the finite mean free path of quarks on photon production by a quark gluon plasma. The main result is that such a correction may have important effects by affecting the collinear sector.

We have first considered the effect of a quark width in the simplest 1-loop diagram, and shown that potentially large 1-loop contributions cancel when a more complete summation is performed. This cancellation is not new in fact, and has been noticed elsewhere. It has connections with the fact that there are no hard thermal loops for vertices with 2 photons and any number of gluons.

Two-loop contributions beyond the eikonal approximation escape this cancellation, and lead to a result which exhibits features of the LPM effect. It is indeed possible to interpret the region where the width of the quark is important as the region where the formation length of the photon is larger than the mean free path of the quark. Our study also shows that the LPM effect modifies the emission spectrum of very hard photons. Despite its nice interpretation, our present result is not complete because it does not consider the vertex corrections that should accompany the resummation of a width.

Multiple scatterings due to longitudinal gluon exchanges are independent, as in the semi-classical treatment of the LPM effect. On the contrary, the transverse sector displays features that are qualitatively different from what is usually accepted in the semi-classical treatment (which just disregards transverse gluon exchanges). Indeed, we find that multiple transverse gluon exchanges are very important also, and that they are not independent due to the long range of magnetic fields.

The various scales in the problem are summarized in Fig. 7. Three of these scales are intrinsic to the quark gluon plasma: the Debye screening length $m_{D}^{-1}$,

the mean free path of the quarks $\lambda_{\text {mean }} \sim \Gamma^{-1}$ and the magnetic screening length $\mu^{-1}$. The fourth scale, the coherence length $\lambda_{\text {coh }}$ (or formation length of the photon), depends on the energy and invariant mass of the photon one wants to observe, and should be compared to the first three. This leads to three zones which have simple physical interpretations. In addition, the nature (and complexity) of the dominant higher loop corrections depend on the coherence length. 


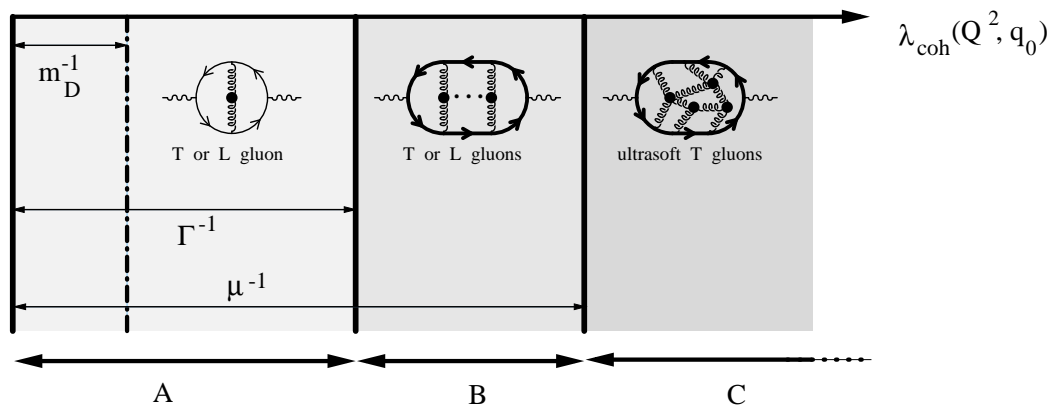

Figure 7: Summary of the various scales in the problem, and comparison with the coherence length. In region A: $0<\lambda_{\text {coh }}<\lambda_{\text {mean }}$. Region $B$ : $\lambda_{\text {mean }}<\lambda_{\text {coh }}<$ $\mu^{-1}$. Region $C: \mu^{-1}<\lambda_{\text {coh }}$. Each picture shows the new class of diagrams that one must consider when going to larger and larger coherence lengths (in addition to the diagrams already considered for smaller coherence lengths). A boldface quark line denotes the resummation of a width in addition to the asymptotic thermal mass.

- Region A: $0<\lambda_{\text {coh }}<\lambda_{\text {mean }}$, and photon production is dominated by single scatterings. The large scale structure of magnetic fields is irrelevant. The only relevant diagram is the 2-loop diagram (with HTL resummed quarks) already considered in [16]. The contributions of transverse and longitudinal gluons are comparable.

- Region B: $\lambda_{\text {mean }}<\lambda_{\text {coh }}<\mu^{-1}$, which implies that photon production is affected by multiple scatterings (LPM effect). In this region, the emission process is not yet sensitive to the magnetic screening. Indeed, according to [18], this sensitivity comes in when $\lambda_{\text {coh }}>\mu^{-1}$. It is sufficient to resum a width (saturated at 1-loop 18) on the quarks, and to consider vertex corrections involving both longitudinal and transverse gluons. So far, we have said nothing about the vertex corrections that should come with self-energy insertions. Nevertheless, it is reasonable to assume from Ward identities that 1-loop self-energy insertions talk to ladder corrections ${ }^{19}$. We can note also that the discussion of this region is somewhat academic, because its extension is only proportional to $\ln (1 / g)$. Additionally, the new topologies of the region $\mathrm{C}$ are at most suppressed by powers of $\ln (1 / g)^{-1}$ if evaluated in region B.

- Region C: $\mu^{-1}<\lambda_{\text {coh }}$. The LPM effect still modifies photon production. In addition, the emission of a photon lasts long enough for the process to be sensitive to the magnetic screening. This is the physical meaning of the result of [18]. Diagrams with an arbitrary number of ultrasoft transverse gluons, connected in

\footnotetext{
${ }^{18}$ When the IR cutoff is $\lambda_{\text {coh }}^{-1} \gg \mu$, corrections to $\Gamma$ coming from topologies beyond 1-loop are suppressed by $g^{2} T / \lambda_{\text {coh }}^{-1} \ll 1$.

${ }^{19}$ The mechanism which makes these ladder corrections important is the same as in 24, 25, and has nothing to do with infrared singularities. The arguments of 18 cannot exclude them, even if the infrared cutoff is $\lambda_{\text {coh }}^{-1} \gg \mu$. This is why these corrections are due to both longitudinal and transverse gluons.
} 
all the possible ways, must be resummed. In addition, the width included on the quarks contains non perturbative contributions due to transverse gluons [22, 23], and the effective gluon vertices must be corrected to hold for ultrasoft momenta 44.

A full study (including vertex corrections) of the corrections to photon production due to longitudinal gluons seems within the reach of perturbation theory, but the situation is very different in the transverse sector which seems far beyond the possibilities of perturbative methods. In this respect, photon production is not very different from the calculation of the quark damping rate [22, 23]. New tools, like functional methods, transport equations, and eventually lattice techiques, are presumably the way to go in this area. In particular, the picture emerging from Fig. 7 suggests an analogy with the successive resummations of 45, 46], in which the scales $T, g T$ and $g^{2} T \ln (1 / g)$ are successively integrated out. Indeed, going to larger and larger coherence lengths requires the inclusion of more and more complicated topologies, a procedure which amounts to integrate out degrees of freedom encountered at smaller length scales.

\section{Acknowledgements}

F.G. would like to thank R. Pisarski, A. Peshier, R. Venugopalan, D. Bödeker and S. Jeon for very interesting comments and discussions. H.Z. thanks the Institute of Nuclear Theory at the University of Washington for its hospitality where part of this work has been done and Dam Thanh Son for fruitful discussions. The work of F.G. is supported by DOE under grant DE-AC0298CH10886.

\section{A Separation of the various cuts}

Equation (27) includes automatically the sum of the cuts $(c)$ and $(d)$ of Fig. 2. To see it explicitly, let us first denote

$$
\begin{aligned}
& F(\Gamma) \equiv \frac{1}{u+\frac{M_{\mathrm{eff}}^{2}}{2 p^{2}}} \\
& G(\Gamma) \equiv \frac{\epsilon(\Gamma)}{\left[\left(u+\frac{M_{\mathrm{eff}}^{2}}{2 p^{2}}+\frac{L^{2}}{2 p^{2}}\right)^{2}-\frac{L^{2}}{p^{2}} \frac{M_{\mathrm{eff}}^{2}}{p^{2}}\right]^{1 / 2}} .
\end{aligned}
$$

Then, the discontinuity on the last line of Eq. (27) equals:

$$
\begin{aligned}
\operatorname{Disc} & F(\Gamma) G(\Gamma)=F(\Gamma) G(\Gamma)-F(-\Gamma) G(-\Gamma) \\
& =\frac{[G(\Gamma)+G(-\Gamma)]}{2} \operatorname{Disc} F(\Gamma)+\frac{[F(\Gamma)+F(-\Gamma)]}{2} \operatorname{Disc} G(\Gamma) .
\end{aligned}
$$

In the last line, the first term corresponds to cut $(d)$ and the second term gives cut $(c)$. The fact that both cuts contribute to the photon rate when $\Gamma \neq 0$ was 
to be expected. Indeed, the cut $(d)$ which corresponds to the process $q \rightarrow q \gamma$ is kinematically forbidden if $\Gamma=0$, but is now allowed by the very fact that the width $\Gamma$ reflects the collisions of the quasi-quark (and the channel $q \rightarrow q \gamma$ is allowed in the presence of a medium).

The integrals to be calculated are more complicated if one wants the two cuts separately. But it turns out that the difference between the two cuts is also very simple. This difference is given by:

$$
\begin{aligned}
(c)- & (d) \propto \frac{[F(\Gamma)+F(-\Gamma)]}{2} \operatorname{Disc} G(\Gamma)-\frac{[G(\Gamma)+G(-\Gamma)]}{2} \operatorname{Disc} F(\Gamma) \\
& =F(-\Gamma) G(\Gamma)-F(\Gamma) G(-\Gamma) .
\end{aligned}
$$

Therefore, in order to get $(c)-(d)$, one has to start from Eq. (27) in which the last line is substituted by $\operatorname{Disc} F(-\Gamma) G(\Gamma)$. The same transformations can be applied to this integral, and one finally obtains an equation similar to Eq. (29), but in which the function $K(w, \widehat{\Gamma})$ is replaced by

$$
L(w, \widehat{\Gamma}) \equiv \frac{1}{2} \int_{0}^{1} \frac{d y}{\sqrt{1-y}} \frac{y+4 / w}{(y+4 / w)^{2}+(1-y)(4 \widehat{\Gamma} / w)^{2}} .
$$

This new integral is also elementary and can be obtained in closed form if needed.

It is obvious that in the limit of zero width $(\widehat{\Gamma} \rightarrow 0)$, the two integrals $K(w, \widehat{\Gamma})$ and $L(w, \widehat{\Gamma})$ become equal. The only way for this to happen is that the contribution of cut $(d)$ goes to zero. Therefore, we recover the fact that the process $q \rightarrow q \gamma$ disappears when $\Gamma=0$.

\section{References}

[1] R.D. Pisarski, Phys. Rev. Let. 63, 1129 (1989).

[2] E. Braaten, R.D. Pisarski, Nucl. Phys. B 337, 569 (1990).

[3] E. Braaten, R.D. Pisarski, Nucl. Phys. B 339, 310 (1990).

[4] J. Frenkel, J.C. Taylor, Nucl. Phys. B 334, 199 (1990).

[5] J. Frenkel, J.C. Taylor, Nucl. Phys. B 374, 156 (1992).

[6] H.A. Weldon, Phys. Rev. D 28, 2007 (1983).

[7] C. Gale, J.I. Kapusta, Nucl. Phys. B 357, 65 (1991).

[8] E. Braaten, R.D. Pisarski, T.C. Yuan, Phys. Rev. Lett. 64, 2242 (1990).

[9] S.M.H. Wong, Z. Phys. C 53, 465 (1992).

[10] J.I. Kapusta, P. Lichard, D. Seibert, Phys. Rev. D 44, 2774 (1991). 
[11] R. Baier, H. Nakkagawa, A. Niegawa, K. Redlich, Z. Phys. C 53, 433 (1992).

[12] P. Aurenche, T. Becherrawy, E. Petitgirard, hep-ph/9403320.

[13] R. Baier, S. Peigné, D. Schiff, Z. Phys. C 62, 337 (1994).

[14] A. Niegawa, Phys. Rev. D 56, 1073 (1997).

[15] P. Aurenche, F. Gelis, R. Kobes, E. Petitgirard, Phys. Rev. D 54, 5274 (1996).

[16] P. Aurenche, F. Gelis, R. Kobes, E. Petitgirard, Z. Phys. C 75, 315 (1997).

[17] P. Aurenche, F. Gelis, R. Kobes, H. Zaraket, Phys. Rev D 58, 085003 (1998).

[18] P. Aurenche, F. Gelis, H. Zaraket, Phys. Rev. D 61, 116001 (2000).

[19] A.D. Linde, Phys. Lett. B 96, 289 (1980).

[20] F. Flechsig, A.K. Rebhan, Nucl. Phys. B 464, 279 (1996).

[21] E. Quack, P.A. Henning, Phys. Rev. Lett. 75, 2811 (1995).

[22] J.P. Blaizot, E. Iancu, Nucl. Phys. B 459, 559 (1996).

[23] J.P. Blaizot, E. Iancu, Phys. Rev. Lett. 76, 3080 (1996).

[24] V.V. Lebedev, A.V. Smilga, Physica A 181, 187 (1992).

[25] V.V. Lebedev, A.V. Smilga, Ann. Phys. (N.Y.) 202, 229 (1990).

[26] M.E. Carrington, R. Kobes, E. Petitgirard, Phys. Rev. D 57, 2631 (1998).

[27] M.E. Carrington, R. Kobes, Phys. Rev. D 57, 6372 (1998).

[28] E. Petitgirard, Phys. Rev. D 59, 045004 (1999).

[29] D. Bodeker, Nucl. Phys. B 566, 402 (2000).

[30] H.A. Weldon, Phys. Rev. D 49, 1579 (1993).

[31] L.D. Landau, I.Ya. Pomeranchuk, Dokl. Akad. Nauk. SSR 92, 535 (1953).

[32] L.D. Landau, I.Ya. Pomeranchuk, Dokl. Akad. Nauk. SSR 92, 735 (1953).

[33] A.B. Migdal, Phys. Rev. 103, 1811 (1956).

[34] R. Baier, Y.L. Dokshitzer, A.H. Mueller, S. Peigné, D. Schiff, Nucl. Phys. B 478, 577 (1996).

[35] B.G. Zakharov, Phys. Atom. Nucl. 61, 838 (1998).

[36] G. Raffelt, D. Seckel, Phys. Rev. Lett. 67, 2605 (1991). 
[37] J. Cleymans, V.V. Goloviznin, K. Redlich, Phys. Rev. D 47, 989 (1993).

[38] D.K. Srivastava, Eur. Phys. J. C 10, 487 (1999).

[39] D.K. Srivastava, B. Sinha, nucl-th/0006018.

[40] M.M. Aggarwal et al., WA98 collaboration, hucl-ex/0006008.

[41] M.M. Aggarwal et al., WA98 collaboration, hucl-ex/0006007.

[42] P. Aurenche, F. Gelis, H. Zaraket, Work in progress.

[43] V.N. Baier, V.M. Katkov, Preprint hep-ph/0002097.

[44] J.P. Blaizot, E. Iancu, Nucl. Phys. B 570, 326 (2000).

[45] D. Bodeker, Nucl. Phys. B 559, 502 (1999).

[46] D. Bodeker, Phys. Lett. B 426, 351 (1998). 